\title{
Automatic Segmentation of Bladder and Prostate Using Coupled 3D Deformable Models
}

\author{
María Jimena Costa*, Hervé Delingette, Sébastien Novellas, \\ and Nicholas Ayache \\ \{Jimena.Costa, Herve.Delingette, Sebastien. Novellas, Nicholas. Ayache\} \\ Qsophia.inria.fr
}

\begin{abstract}
In this paper, we propose a fully automatic method for the coupled 3D localization and segmentation of lower abdomen structures. We apply it to the joint segmentation of the prostate and bladder in a database of CT scans of the lower abdomen of male patients. A flexible approach on the bladder allows the process to easily adapt to high shape variation and to intensity inhomogeneities that would be hard to characterize (due, for example, to the level of contrast agent that is present). On the other hand, a statistical shape prior is enforced on the prostate. We also propose an adaptive non-overlapping constraint that arbitrates the evolution of both structures based on the availability of strong image data at their common boundary. The method has been tested on a database of 16 volumetric images, and the validation process includes an assessment of inter-expert variability in prostate delineation, with promising results.
\end{abstract}

Keywords: prostate, bladder, 3D segmentation, coupled deformable models, CT.

\section{Introduction}

An essential part of a successful conformal radiotherapy treatment planning procedure is the accurate contouring of target volumes and organs at risk.

Because of the difficulty to accurately and reliably delineate structures in medical images, this task has traditionally been assigned to medical experts. However, manual editing is not only tedious but particularly prone to errors.

Semi-automatic or interactive approaches for segmentation allow the pratician to have better control over the segmentation process [1,2]. However, they remain time consuming and, especially for large databases, an automatic approach is desirable.

The segmentation of pelvic structures is a particularly difficult task since it involves soft tissues that present a very large variability in shape, size and intensity, the latter depending on the presence (partial or total) or absence of a contrast agent. The task is even more challenging in the case of prostate cancer, since, in this case, the characteristics of the organs at risk (bladder, rectum) are

\footnotetext{
^ www-sop.inria.fr/asclepios/personnel/Jimena.Costa/
} 
highly variable and have an important influence on the shape and location of the target organ itself (prostate).

After a short review of previous work, we present a novel framework for the localization and coupled segmentation of the prostate and bladder in CT images.

\section{Previous Work}

\subsection{Un-coupled Bladder and Prostate Segmentation}

Registration Approaches. These methods have been tested for CT bladder [3] and prostate [4] segmentation. Heavy variations in soft tissue (shape, size, intensity) are difficult to capture by these approaches, but they remain quite useful for initialization purposes.

Mathematical Morphology Approaches. Variations of these approaches have been tested in [5, 6. They are easy to automate and can be quickly tuned and computed, but they are strongly dependent on the quality of the image.

Shape Deformation Approaches. Deformable models are quite flexible, since they can include shape priors [7,1,8], atlas initialization [9], fuzzy criteria [10] and multiple structure deformation [7. Explicit models have been used for both prostate 11, 12 and bladder 10] segmentation. Implicit models have also been used to this end (see 8 for prostate and [13 for bladder segmentation).

Other Approaches. Other approaches include neural networks [2, radial searching [14, polar transform based methods [15] and genetic algorithms [16. 17], among others.

\subsection{Multiple Structure Segmentation}

Overlap penalization is proposed for both explicit [18] and implicit [7] models. In both cases, overlaps are punished in terms of energy minimization. The first approach couples multiple active contours in $2 \mathrm{D}$ video sequences through a unique energy function. The second presents a Bayesian inference framework where a shape prior can be applied on any of the structures. Neither approach is able to handle non-characterizable intensity inhomogeneities within structures.

Statistical shape and appearance model approaches are used in [11, 19, 20, 21,22, among others. In [20, a segmentation method using both an intensity prior based on intensity profiles at each point and a geometric typicality (shape prior) is proposed. In [19,21,22, a perfect partition of the image into classes of similar intensities or textures is achieved. However, these techniques treat objects sharing similar image characteristics as a single item; thus, topological constraints between them cannot be enforced in the absence of a clear delimitation of the structures (for example, in a non-contrasted bladder and prostate case). 
In fact, the approaches that we have found in the literature impose quite strong shape and/or appearance constraints on the structures involved.

The methods are therefore restricted to homogenous structures, or structures with characterizable inhomogeneities, which is not always the case in our database of images. We are thus motivated to propose a fully automatic framework for coupled bladder-prostate segmentation using explicit deformable models and a non-overlapping constraint. The method adjusts itself to different kinds of bladder (homogenous, different levels of inhomogeneities). The characteristics of the interface between both structures in the image are taken into account in this constraint. Since the prostate shows a much better statistical coherency in shape among patients than the bladder ( [7]), a prostate shape prior is enforced.

\section{Our Approach to Bladder Segmentation}

In order to put the CT images in a common reference frame, locally affine registration 23] is performed on the pelvic bone structures (since they show lower variability than smooth tissues) and then interpolated to the soft tissues in the image. This allows us to perform the same cropping process on all the images, and to have a first localization of the target organs.
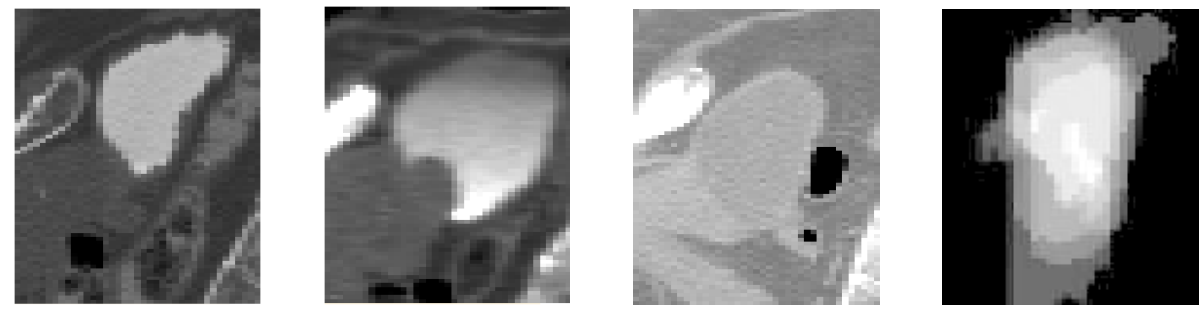

Fig. 1. Different types of bladders make the segmentation task challenging. From left to right, homogenous contrasted bladder, non-homogenous bladder and homogenous non-contrasted bladder (sagittal views). The rightmost image shows the variability in bladder shape and size, even in registered images (sagittal view).

The bladder is first located and classified as homogenous or non-homogenous, contrasted or non-contrasted (Figure 1) using a modified version of the region growing approach with highly contrasted voxels located within a zone of low intensity variability as seed points. Then, the segmentation begins by computing an approximation of the structure through mathematical morphology operations. A simplex mesh is deformed to fit this approximation. If the structure is not homogenous, the model is divided into zones that correspond to those found in the image data. The segmentation is later refined and smoothed using the bladder in the CT image itself. This process is illustrated in Figure 2 A more detailed explanation and validation of this method was presented in [24]. 

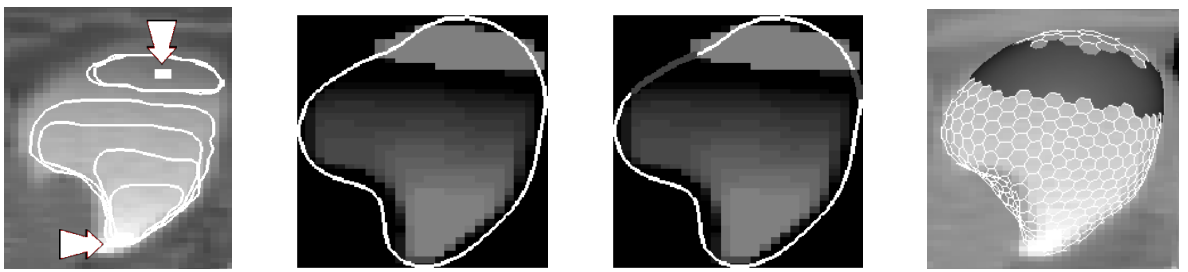

Fig. 2. Progression of modified region growing algorithms for a non-homogenous bladder, in order to generate a binary approximation of the structure. Seed points are indicated by arrows (left). An initial model is then deformed over the resulting approximation, and eventually divided into zones (if required by structure intensity inhomogeneities). The segmentation is then refined using the original, grey level image.

\section{Our Approach to Prostate Segmentation}

\subsection{Prostate Localization}

We use prior information on prostate localization, which has been computed based on the expert's segmentations of the structure in our database images. These images had previously been placed in a common frame of reference for bladder segmentation purposes using a non-rigid registration approach (see section 3).

\subsection{Shape Statistics}

The shape of the prostate across large patient population shows statistical coherency [7, but the image data is often not sufficient to establish the outline of this structure, it is helpful to incorporate shape prior knowledge. We built a shape model of the prostate from a database of training samples (CT images and their corresponding segmentations of the prostate performed by an expert). An initial deformable model was used to fit the manual segmentations of the prostates in the database, thus assuring a point correspondence between the models. A mean shape model and its principal deformation modes were computed using a Principal Component Analysis approach.

\subsection{Intensity Information}

We obtain initial information about the intensity of the prostate in each image from a small region inside the target structure. We define this region around a starting point located inside the mean shape model. We choose, among all the potential starting points, one located in a neighbourhood showing little intensity variance within a previously computed interval in Hounsfield units.

\subsection{Initial Prostate Model Deformation}

At each time step $t$, the position of vertex $V_{i}$ in the prostate model is computed according to Equation 1.

$$
V_{i}^{t+1}=V_{i}^{t}+\lambda\left(\alpha\left(f_{i}^{P C A}\right)+\beta\left(f_{i}^{e x t}\right)+\delta\left(f_{i}^{i n t}\right)\right)+(1-\lambda)\left(f_{i}^{\text {global }}\right)
$$


where $V_{i}^{t}$ and $V_{i}^{t+1}$ are the positions of vertex $i$ at time $t$ and $t+1$, respectively. Parameter $\lambda$ is a locality parameter: we start with $\lambda=0$, a purely global (rigid + affine) deformation, and move progressively towards a more local deformation $(0<\lambda<1)$. The influences of $f^{P C A}$ (the PCA-based regularization force), $f^{e x t}$ (an image force that enforces intensity homogeneity within the structure) and $f^{\text {int }}$ (internal regularization force) are weighted by parameters $\alpha, \beta$ and $\delta$, respectively. Their values have been set to $0.4,0.3$ and 0.3 .

The force $f^{P C A}$ pulls the current model $\mathcal{S}$ towards $\mathcal{S}_{\mathrm{S}}$, a "smooth" surface that belongs to the space spanned by the computed PCA modes of variation (for regularization purposes) and $f^{\text {ext }}$ pulls it towards $\mathcal{S}_{\text {I }}$, an estimated target surface corresponding to the boundaries of the anatomical structure in the image. If we assume that the normal $\mathbf{n}(\mathbf{u})$ to the current surface $\mathcal{S}(\mathbf{u})$ is oriented outwards, the image guided term $\mathcal{S}_{\mathrm{I}}(\mathbf{u})$ can be computed at each iteration as $\mathcal{S}(\mathbf{u})+s_{\star} \mathbf{n}$ as

$$
s_{\star}=\arg \min _{s \in[-L ; L]} \sum_{v=-L}^{v=L} G_{\sigma}(|v-s|) * f(\mathcal{I}(\mathcal{S}(\mathbf{u})+v \mathbf{n}), \mu, \sigma, \operatorname{sgn}(v-s))
$$

where $s$ is the position of each vertex of the final mesh we want to evaluate, $-L$ and $L$ are bounds on $s, v$ is the position of the voxels along the normal of the mesh at vertex $s$, and $f(i, \mu, \sigma, s g n)$ is a confidence estimation. This confidence is a piecewise constant function that serves to increase or decrease the energy term, depending on the values of two expressions: $\left|\frac{\mathcal{I}(\mathcal{S}(\mathbf{u})-\mu}{\sigma}\right| \leq 2$ and $\operatorname{sgn}(v-s)$. For example, if the first term is false (i.e., the voxel's intensity is not compatible with the intensities found inside the structure) and the second term is true (i.e. the voxel is located inside the mesh), a positive penalization value is added to the energy term. Function $G_{\sigma}$ defines a weight for the voxels that are taken into account at each iteration step; it may be a Gaussian p.d.f., a generalized rectangle function, or a combination of the two. The parameters are fully adjustable, to penalize more (or less) a non-homogeneity inside the structure or zone.

Once this strict deformation process stabilizes, we bring prostate-bladder model interaction into the game.

\section{Context-Dependent Coupled Deformation}

We present a coupled segmentation framework in which an asymmetric, nonoverlapping constraint is enforced.

The non-overlapping of the structure models is achieved through the use of a specifically designed force to each mesh in the coupled deformation process (inspired by ( [25])). At each deformation step, the areas enclosed by both the prostate and bladder models are checked for intersection. If such an intersection exists, a new elastic force, proportional to the distance maps to the meshes, is added to both models in order to drive them appart.

If the prostate-bladder interface is sufficiently "clear" (as in the case of contrasted bladders), we apply a symmetric non-overlapping force to both models. 

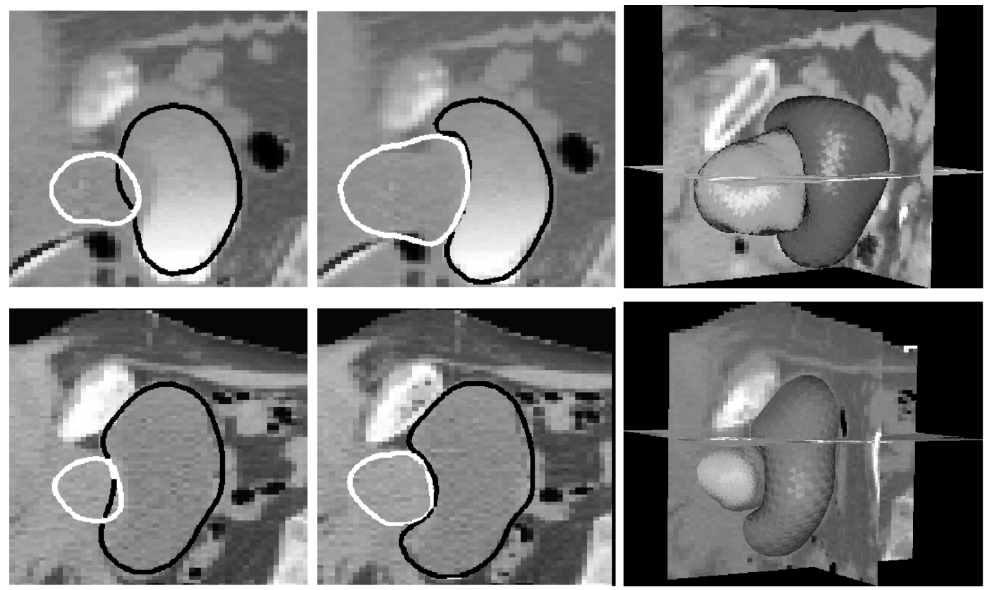

Fig. 3. Examples of the effect of the non-overlapping constraint in both partially clear (first row) and fuzzy (second row) prostate-bladder interfaces. From left to right, the independent evolution of prostate and bladder models, their coupled evolution with our non-overlapping constraint, and a $3 \mathrm{D}$ view of the result.

If, on the other hand, the interface is blurry, a higher priority is given to the model that contains the most information (like a shape prior), which is, in our case, the prostate mesh.

Our proposed asymmetric, context dependent non-overlapping constraint comprises interaction forces between the prostate and bladder meshes $\left(F_{P o B}\right.$ and $\left.F_{B o P}\right)$. To this end, the prostate shape model, the bladder model $\left(F_{B o P}\right)$ and the strength of the border between the two in the image $(f(\|\nabla I\|))$ are taken into account, as shown by equations 3 and 4

$$
\begin{gathered}
F_{B o P}=-\eta_{P} *\left(\left(\nabla \operatorname{Dmap}_{B}\right) /\left\|\nabla \operatorname{Dmap}_{B}\right\|\right) *\left(\operatorname{Dmap}_{B}-\tau_{B}\right) * f(\|\nabla I\|) \\
F_{P o B}=-\eta_{B} *\left(\left(\nabla \operatorname{Dmap}_{P}\right) /\left\|\nabla \operatorname{Dmap}_{P}\right\|\right) *\left(\operatorname{Dmap}_{P}-\tau_{P}\right) *(1-f(\|\nabla I\|))
\end{gathered}
$$

where

$$
f(\|\nabla I\|)= \begin{cases}\frac{\|\nabla I\|}{\vartheta}, & \text { if }\|\nabla I\| \leq \vartheta \text { (i.e., zone with low contrast) } \\ 1, & \text { if }\|\nabla I\|>\vartheta \text { (i.e., zone with high contrast) }\end{cases}
$$

Parameters $\eta_{P}$ and $\eta_{B}$ weight the strength of the repulsion force, $\operatorname{Dmap}_{B}$ and $\operatorname{Dmap}_{P}$ are the distance maps to the bladder and prostate models, respectively, $\tau_{B}$ and $\tau_{P}$ are thresholds chosen on the distance maps (to establish a minimum distance between the models, if desired), $\|\nabla I\|$ is the norm of the image gradient, and $\vartheta$ is a threshold on the image gradient value to distinguish contrasted from non-contrasted prostate-bladder interfaces. 


\section{$6 \quad$ Results and Perspectives}

The automatic segmentation algorithm was applied to a database of $16 \mathrm{CT}$ images of the lower abdomen of male patients. The results were compared to experts' segmentations of bladder and prostate (the manual segmentation sets used for training and validation are disjoint). Figure 4 shows the obtained sensitivities and positive predictive values: the average sensitivity / positive predictive value is $0.81 / 0.85$ for the bladder, and $0.75 / 0.80$ for the prostate.

\begin{tabular}{|c|cc|cc|}
\hline & \multicolumn{2}{|c|}{ Bladder } & \multicolumn{2}{c|}{ Prostate } \\
Image & \multicolumn{2}{|c|}{ Sensit. PPV } & Sensit. PPV \\
\hline 1 & 0.82 & 0.94 & 0.73 & 0.99 \\
2 & 0.87 & 0.95 & 0.86 & 0.92 \\
3 & 0.88 & 0.94 & 0.75 & 0.91 \\
4 & 0.86 & 0.89 & 0.95 & 0.72 \\
5 & 0.89 & 0.97 & 0.72 & 0.81 \\
6 & 0.86 & 0.86 & 0.79 & 0.81 \\
7 & 0.91 & 0.96 & 0.86 & 0.87 \\
8 & 0.87 & 0.85 & 0.82 & 0.94 \\
\hline
\end{tabular}

\begin{tabular}{|c|cc|cc|}
\hline & \multicolumn{2}{|c|}{ Bladder } & \multicolumn{2}{c|}{ Prostate } \\
Image & Sensit. PPV & Sensit. PPV \\
\hline 9 & 0.87 & 0.93 & 0.76 & 0.84 \\
10 & 0.88 & 0.84 & 0.85 & 0.84 \\
11 & 0.91 & 0.93 & 0.80 & 0.89 \\
12 & 0.82 & 0.77 & 0.72 & 0.89 \\
13 & 0.69 & 0.92 & 0.79 & 0.64 \\
14 & 0.82 & 0.79 & 0.89 & 0.82 \\
15 & 0.94 & 0.97 & 0.79 & 0.90 \\
16 & 0.85 & 0.97 & 0.74 & 0.77 \\
\hline
\end{tabular}

Fig. 4. Sensitivity and Positive Predictive Value results of the automatic segmentation of the bladder (left) and prostate (right) with respect to the one performed by an expert

For the validation of the prostate segmentations, we were able to assess the inter-expert variability thanks to a database of 5 CT images in which the prostates had been segmented by 3 different experts. We used the STAPLE [26] algorithm to compute a mean expert segmentation, and compared both the manual (expert) and automatic segmentations with respect to this mean. The results (Figure 5) show that the automatic segmentations are not far from the ones performed by the experts.

The results are promising, in spite of the low saliency (sometimes even indistinguishability) of the prostate in the images. The bladder-prostate interface

\begin{tabular}{|c|c|c|c|c|c|c|c|c|}
\hline \multirow[b]{2}{*}{ Image } & \multicolumn{2}{|c|}{ Expert 1} & \multicolumn{2}{|c|}{ Expert 2} & \multicolumn{2}{|c|}{ Expert 3} & \multicolumn{2}{|c|}{ Automatic } \\
\hline & Sensit & PPV & Sensi & PPV & Sensi & PPV & Sen: & PPV \\
\hline 1 & 0.82 & 0.87 & 0.98 & 0.80 & 0.94 & 0.91 & 0.71 & 0.98 \\
\hline 5 & 0.80 & 0.99 & 0.99 & 0.64 & 0.90 & 0.96 & 0.72 & 0.85 \\
\hline 6 & 0.82 & 0.89 & 0.96 & 0.75 & 0.99 & 0.97 & 0.81 & 0.81 \\
\hline 9 & 0.92 & 0.95 & 0.98 & 0.69 & 0.77 & 0.98 & 0.66 & 0.92 \\
\hline 10 & 0.91 & 0.91 & 0.96 & 0.96 & 0.97 & 0.84 & 0.89 & 0.79 \\
\hline
\end{tabular}

Fig. 5. Sensitivity and Positive Predictive Value of both the expert and automatic segmentations of the prostate, with respect to the computed mean expert segmentation using the STAPLE [26] algorithm. 
is correctly found. As the experts have confirmed, the prostate border that is not shared with the bladder is difficult to delineate, since there is little or no image information in this zone. This introduces some variability in the figures. We will continue to investigate this, and we would also like to incorporate multisequence analysis with MR and CT images, in order to have a better visibility of the prostate. Eventually, we will include the rectum in the joint segmentation process, since it is also an organ at risk during prostate cancer radiotherapy.

The work described in this article was performed in collaboration with Dosisoft, in the framework of the European Integrated Project MAESTRO, funded by the European Commission.

\section{References}

1. Freedman, D., Zhang, T.: Interactive graph cut based segmentation with shape priors. In: CVPR 2005, vol. 1, pp. 755-762. IEEE, Washington, DC, USA (2005)

2. Lee, C., Chung, P.: Identifying abdominal organs using robust fuzzy inference model. In: ICNSC 2004, vol. 2, pp. 1289-1294. IEEE, Washington, DC, USA (2004)

3. Unal, G., Slabaugh, G.: Coupled pdes for non-rigid registration and segmentation. In: CVPR 2005, vol. 1, pp. 168-175. IEEE, Washington, DC, USA (2005)

4. Malsch, U., Thieke, C., Bendl, R.: Fast elastic registration for adaptive radiotherapy. In: Larsen, R., Nielsen, M., Sporring, J. (eds.) MICCAI 2006. LNCS, vol. 4191, pp. 612-619. Springer, Heidelberg (2006)

5. Camapum, J., Silva, A., Freitas, A., et al.: Segmentation of clinical structures from images of the human pelvic area. In: SIBGRAPI 2004, pp. 10-16. IEEE Computer Society, Washington, DC, USA (2004)

6. Mazonakis, M., Damilakis, J., Varveris, H., et al.: Image segmentation in treatment planning for prostate cancer using the region growing technique. Br. J. Radiol. 74(879), 243-248 (2001)

7. Rousson, M., Khamene, A., Diallo, M., et al.: Constrained surface evolutions for prostate and bladder segmentation in ct images. In: Liu, Y., Jiang, T., Zhang, C. (eds.) CVBIA 2005. LNCS, vol. 3765, pp. 251-260. Springer, Heidelberg (2005)

8. Broadhurst, R., Stough, J., Pizer, S., et al.: Histogram statistics of local modelrelative image regions. In: Olsen, O.F., Florack, L.M.J., Kuijper, A. (eds.) DSSCV 2005. LNCS, vol. 3753, pp. 72-83. Springer, Heidelberg (2005)

9. Ripoche, X., Atif, J., Osorio, A.: A 3d discrete deformable model guided by mutual information for medical image segmentation. In: Proceedings of the Medical Imaging Conference 2004, SPIE (2004)

10. Bueno, G., Martínez-Albalá, A., Adán, A.: Fuzzy-snake segmentation of anatomical structures applied to ct images. In: ICIAR (2), pp. 33-42 (2004)

11. Freedman, D., Radke, R., Zhang, T., et al.: Model-based multi-object segmentation via distribution matching. In: CVPRW 2004, vol. 1, p. 11. IEEE Computer Society, Washington, DC, USA (2004)

12. Dam, E., Fletcher, P.T., et al.: Prostate shape modeling based on principal geodesic analysis bootstrapping. In: Barillot, C., Haynor, D.R., Hellier, P. (eds.) MICCAI 2004. LNCS, vol. 3217, pp. 1008-1016. Springer, Heidelberg (2004)

13. Tsai, A., Yezzi, A.J., et al.: A shape-based approach to the segmentation of medical imagery using level sets. IEEE TMI 22(2), 137-154 (2003) 
14. Xu, W., Amin, S., Haas, O., et al.: Contour detection by using radial searching for ct images. In: 4th EMBS Special Topic Conference, pp. 346-349. IEEE Computer Society, Washington, DC, USA (2003)

15. Zwiggelaar, R., Zhu, Y., et al.: Semi-automatic segmentation of the prostate. In: IbPRIA pp. 1108-1116 (2003)

16. Cosío, F.A.: Prostate segmentation using pixel classification and genetic algorithms. In: MICAI, pp. 910-917 (2005)

17. Ghosh, P., Mitchell, M.: Segmentation of medical images using a genetic algorithm. In: GECCO 2006, pp. 1171-1178. ACM Press, New York (2006)

18. Zimmer, C., Olivo-Marin, J.C.: Coupled parametric active contours. IEEE Trans. Pattern Anal. Mach. Intell. 27(11), 1838-1842 (2005)

19. Paragios, N., Deriche, R.: Geodesic active regions: a new paradigm to deal with frame partition problems in computer vision. JVCIR 13(1/2), 249-268 (2002)

20. Pizer, S.M., Fletcher, P.T., Joshi, S., Gash, A.G., Stough, J., Thall, A., Tracton, G., Chaney, E.L.: A method and software for segmentation of anatomic object ensembles by deformable m-reps. Med. Phys. 32(5), 1335-1345 (2005)

21. Vese, L.A., Chan, T.F.: A multiphase level set framework for image segmentation using the mumford and shah model. Int. J. Comput. Vision 50(3), 271-293 (2002)

22. Yezzi Jr, A., Tsai, A., Willsky, A.: A fully global approach to image segmentation via coupled curve evolution equations. JVCIR 13(1/2), 195-216 (2002)

23. Commowick, O., Arsigny, V., Costa, J., et al.: An efficient locally affine framework for the registration of anatomical structures. In: ISBI 2006, Arlington, Virginia, USA (2006)

24. Costa, M., Delingette, H., Ayache, N.: Automatic segmentation of the bladder using deformable models. In: ISBI 2007, Arlington, Virginia, USA (2007)

25. Pitiot, A., Delingette, H., Thompson, P.M., Ayache, N.: Expert knowledge guided segmentation system for brain MRI. NeuroImage 23(supplement 1), S85-S96 (2004) Special Issue: Mathematics in Brain Imaging

26. Warfield, S.K., Zou, K.H., Wells. III, W.M.: Simultaneous truth and performance level estimation (staple): an algorithm for the validation of image segmentation. IEEE TMI 23(7), 903-921 (2004) 